NOTICE : this is the author's version of a work that was accepted for publication in Composites Science and Technology. Changes resulting from the publishing process, such as peer review, editing, corrections, structural formatting, and other quality control mechanisms may not be reflected in this document. Changes may have been made to this work since it was submitted for publication. A definitive version was subsequently published in Composites Science and Technology (2006), doi:10.1016/j.compscitech.2006.02.037.

\title{
Two percolation thresholds in carbon nanotube epoxy composites
}

\author{
Josef Z. Kovacs ${ }^{a,}{ }^{,}$, Bala S. Velagala ${ }^{a}$, Karl Schulte ${ }^{b}$, Wolfgang Bauhofer ${ }^{a}$ \\ ${ }^{a}$ Institut für Optische und Elektronische Materialien, Technische Universität Hamburg-Harburg, D-21073 \\ Hamburg, Germany \\ ${ }^{b}$ Polymer Composites Section, Technische Universität Hamburg-Harburg, D-21073 Hamburg, Germany
}

\begin{abstract}
The results presented in this paper reveal the first evidence that two types of percolation thresholds can coexist in an insulator-conductor-system, the higher one attributed to a static and the lower one to a kinetic network formation process. Detailed measurements at concentrations above the statistical percolation threshold show a power law dependence of conductivity on filler concentration (with an exponent of 2.7) that is independent of the processing condition. A simplified model of percolated particles was developed in order to relate the sample conductivity to the contact resistance between individual particles embedded within a polymer matrix. Our results are compared with previous studies in order to correlate the contact resistance values to different materials and processing conditions.
\end{abstract}

Keywords: Carbon nanotubes; Epoxy matrix; Electrical properties; Percolation; Network formation

\section{Introduction}

Conductive filler particles in an insulating matrix are able to lower the overall resistivity by several orders of magnitude when a network develops throughout the matrix. The transition from an insulating to a conducting composite as a function of filler concentration is known as percolation and the critical concentration at which this drop occurs is called percolation threshold.

Percolation is described by many theories using different approaches. Some of them take into account the dependence of the percolation threshold on the filler shape [1], size [2] or aspect ratio [3], based upon an excluded volume approach [4] of infinitely thin particles. Another approach considers the dependency on the orientation of finitely thick sticks [5]. These statistical theories generally assume a random filler particle distribution

* Corresponding author. Tel.: +49 4042878 3853; Fax: +49 4042878 2229. E-mail address: josef.kovacs@tuhh.de (J.Z. Kovacs). 
and furthermore are static theories because they do not include the possibility of particle movement through the matrix. They predict percolation thresholds that are orders of magnitude higher than found in many experiments [6-8]. All these experiments have in common that they were performed on systems where the matrix possesses a fluid state of low viscosity (< 1 Pas) during processing. In this state particle manipulation is possible via shear forces $[7,8]$ and electric fields [9] which promote the formation of a network at filler contents as low as 0.002 wt\% [8]. Such low percolation thresholds are no longer static but dynamic and have to be described by dynamic colloid theory [10].

This work investigates the effect of shear rates on the promotion of a particle network at concentrations below and above the statistical percolation threshold. The system under test consists of multi-wall carbon nanotubes dispersed in an epoxy resin which possesses a fluid state of low viscosity during processing. Variable shear rates are exerted by controlling the stirring rate of the agitator and the temperature of the dispersion during the final mixing step.

However, an existing network does not guarantee good conductivity if the contact resistance between the individual filler particles is too high. Therefore, a simple relation between sample conductivity and filler content was derived for the region above the static percolation threshold in order to estimate the magnitude of the contact resistance between individual particles embedded within a polymer matrix. These calculations were also applied to conductivity measurements published by other groups [11-15] in order to correlate the contact resistance values to different polymer materials and processing conditions.

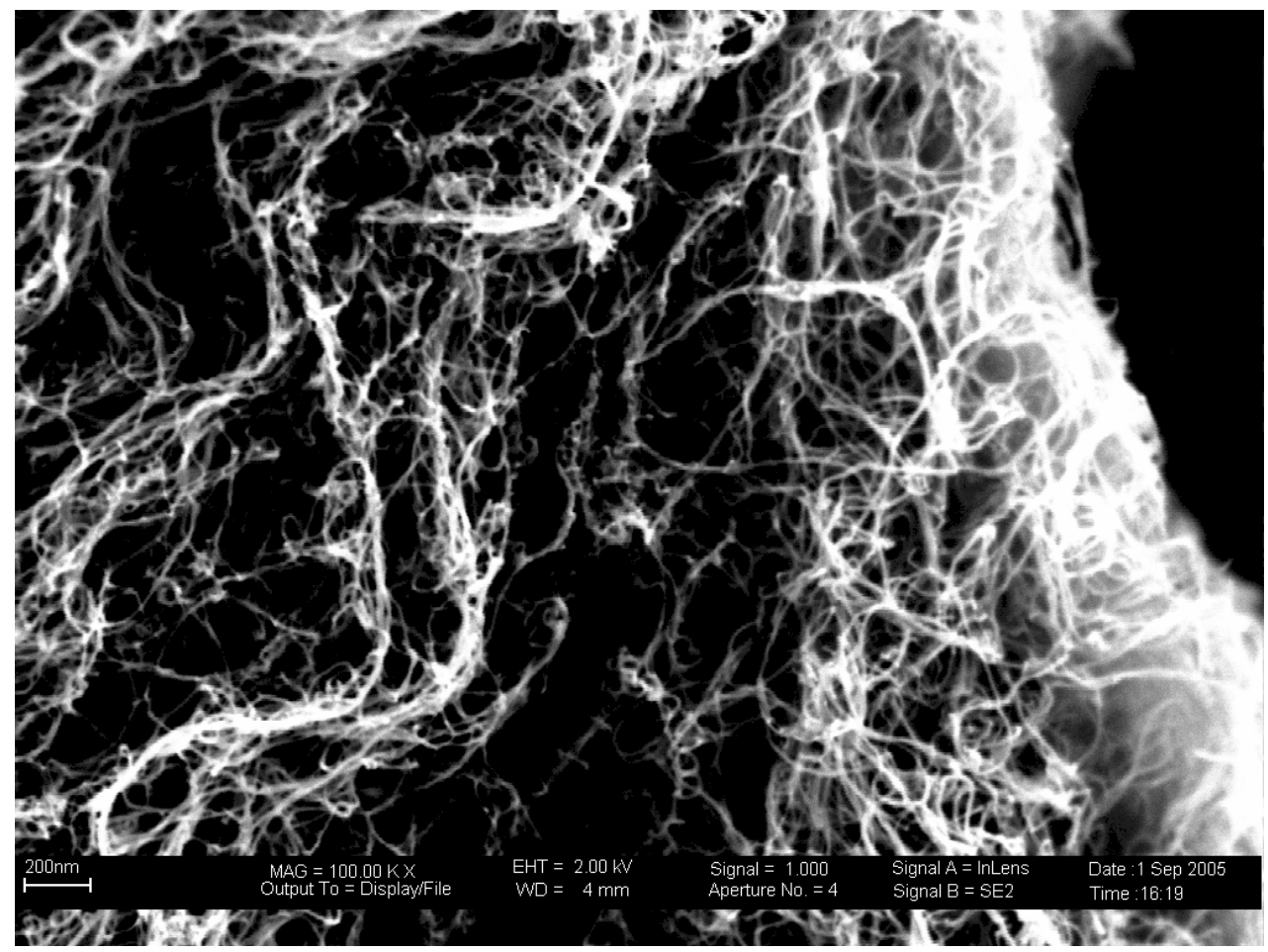

Fig. 1. SEM-image of a cluster of CCVD-grown multi-wall carbon nanotubes as supplied. The scale bar is $200 \mathrm{~nm}$. 


\section{Materials and experimental details}

Multi-wall carbon nanotubes grown by catalytic chemical vapor deposition (CCVD) were supplied by Nanocyl S.A. (Belgium) specified with average inner and outer diameter of 4 and $15 \mathrm{~nm}$, respectively, lengths up to $50 \mu \mathrm{m}$ and carbon purity exceeding $95 \%(<5 \%$ iron catalyst). The scanning electron microscopy image (Fig. 1) shows a typical cluster of as-received nanotubes, which had to be broken up via shearing. This procedure tends to fracture the nanotubes due to their entanglement, thus an average length of several $\mu \mathrm{m}$ was assumed throughout our experiments. The polymer matrix consisted of bisphenol-A-based epoxy resin (Araldite LY 556) with an amine-based hardener (XB 3473), obtained from Huntsman Advanced Materials (Belgium).

Samples of nanotube epoxy composites of varying filler concentrations were produced in the following way. A masterbatch of epoxy resin containing a high nanotube concentration was prepared with a dissolver disk rotating at $2000 \mathrm{rpm}$ for 2 hours at room temperature. Approximately $30 \mathrm{~g}$ of this dispersion was poured into a small cup, the appropriate amount of hardener (mixing ratio of 23:100 parts per weight of hardener:resin) was added and subsequently processed in three different ways. The filler concentration in the remaining masterbatch was then lowered adding pure resin and again stirred for 30 minutes at $2000 \mathrm{rpm}$. A suspension of $30 \mathrm{~g}$ was again extracted, and the procedure described above was repeated several times. The first sample set was stirred at $500 \mathrm{rpm}$ for 10 minutes at $80{ }^{\circ} \mathrm{C}$, then poured into aluminium moulds and further stirred at $50 \mathrm{rpm}$ for $5 \mathrm{~min}$ at $80{ }^{\circ} \mathrm{C}$ (in the following referred to as the sample set with slow stirring, thus SS). The second set of samples was only stirred at $500 \mathrm{rpm}$ for 10 min at $80{ }^{\circ} \mathrm{C}$ and afterwards left untouched in the aluminium moulds for 5 more minutes (sample with medium stirring, MS). The third sample set was stirred at 2000 rpm for $15 \mathrm{~min}$ at room temperature (sample with fast stirring, FS) and then poured into aluminium moulds. This set of samples was further equipped with brass electrodes (17 $\mathrm{mm}$ width, clamped in $1.8 \mathrm{~mm}$ distance and dipped $10 \mathrm{~mm}$ deep into each portion) in order to monitor conductivity while curing. All samples were cured in an oven at $120{ }^{\circ} \mathrm{C}$.

After curing, two specimens $(8 \times 18 \times 4 \mathrm{~mm})$ were cut out from each sample with SS and MS processing. The $8 \times 18 \mathrm{~mm}$ sides were polished, coated with conductive silver paint and contacted for conductivity measurements. The embedded electrodes were used as contacts for the FS-samples. Electrical measurements were performed in AC mode using a HP 4284A LCR meter at room temperature with a voltage amplitude of $1.4 \mathrm{~V}$ over a frequency range from $20 \mathrm{~Hz}$ to $1 \mathrm{MHz}$. The $\mathrm{AC}$ conductivities were calculated from the admittance values and, being constant up to at least $1 \mathrm{kHz}$, the values at 100 $\mathrm{Hz}$ were considered to be DC-equivalent. For optical analysis slices were cut out from all three sample sets and polished down to a thickness of only $0.5 \mathrm{~mm}$. 


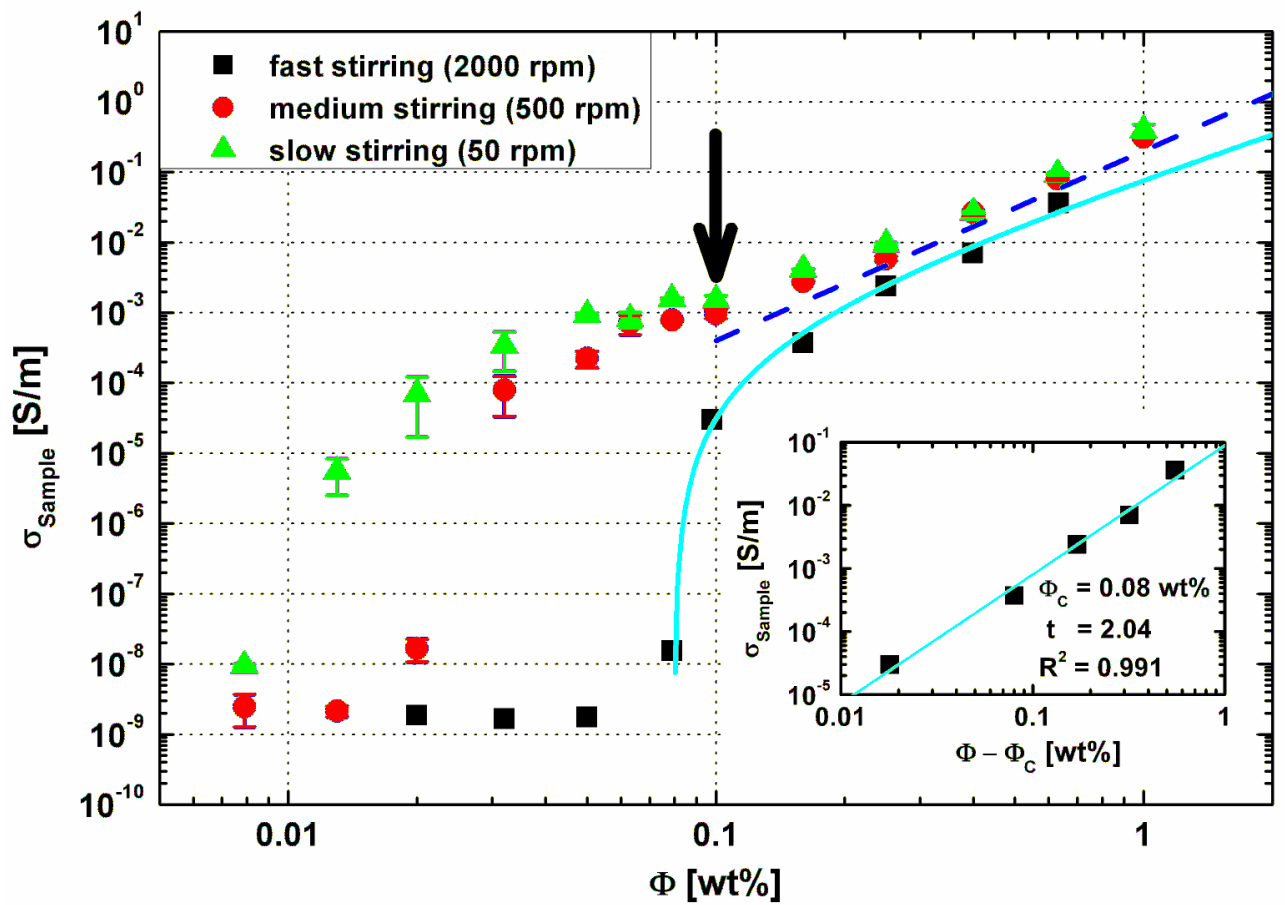

Fig. 2. Comparative log-log plot of the nanocomposite conductivity as a function of nanotube weight fraction for the three different sample preparation methods. The arrow denotes the slope crossover, the dashed line illustrates the dependence $\sigma_{\text {Sample }} \sim \Phi^{2.7}$ and the inset represents $\log \sigma_{\text {Sample }} v s$ $\log \left(\Phi-\Phi_{C}\right)$ with the best fit to the data of the FS-samples (solid line with slope $t=2.04$ ).

\section{Results}

The conductivity values of all samples as a function of nanotube concentration are summarized in a log-log plot in Fig. 2. The values for the lowest concentrations correspond to the pure epoxy resin conductivity of $10^{-9} \mathrm{~S} / \mathrm{m}$. For the SS-samples the percolation threshold is approximately $0.011 \mathrm{wt} \%$ while for the MS-samples it is around $0.024 \mathrm{wt} \%$. These values are comparable with the threshold found in previous experiments $(0.03 \mathrm{wt} \%)$ performed with similarly entangled nanotubes [6] but are one order of magnitude higher than achievable with aligned nanotubes [7]. As we will argue in chapter 5, these percolation thresholds are due to kinetic processes and therefore cannot be determined using the common percolation scaling law from statistical percolation theory [16,17]. Therefore, our thresholds were taken at half of the conductivity increase from pure epoxy level to the incipient plateau at $10^{-3} \mathrm{~S} / \mathrm{m}$. This conductivity plateau develops for both sets and sustains until $0.1 \mathrm{wt} \%$. Here, a crossover from saturation to a power law behavior is clearly visible (arrow in Fig. 2). While investigations of Sandler et al. [7] on a similar system already suggest both, the plateau and the onset of a slope crossover in their conductivity plot, unambiguous evidence was provided through our measurements for the first time. This was made possible by the large number of measurements over a wide range of concentrations and the smaller increments of concentration than previously considered. 
The experimental values of the FS-samples are well fitted by the scaling law $\sigma \sim\left(\Phi-\Phi_{C}\right)^{t}$ with critical concentration $\Phi_{C}=0.08$ wt $\%$ and exponent $t=2.04$ (solid lines in both, Fig. 2 and its inset). The critical exponent is in good agreement with predictions from statistical percolation theory for a three-dimensional conducting network in an insulating matrix [17] just as with other experimental results $[11,15]$. However, for rodlike particles lower [18] as well as higher [14,19] values can be found in literature. It should be noted that the conductivity of the FS-samples follows - above $0.1 \mathrm{wt} \%$ - the power law $\sigma_{\text {Sample }} \sim \Phi^{2.7}$ just like the SS- and MS-samples.

Light microscopy images of thin slices cut from cured samples are shown in Fig. 3. For each preparation method the emergence of flocs and the formation of a kind of superstructure are detectable. It is obvious that they both appear at the same concentrations where the conductivity increases by several orders of magnitude. However, above $0.2 \mathrm{wt} \%$ all samples exhibit a homogeneous particle distribution as exemplified in the last column of Fig. 3.

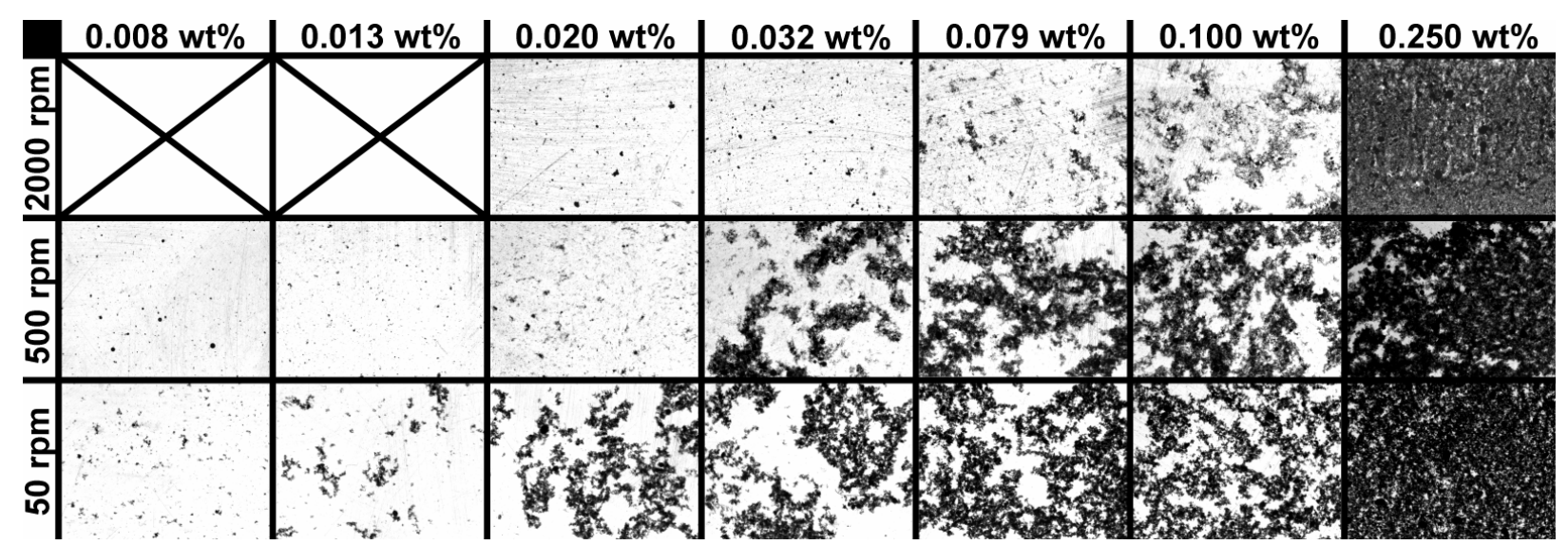

Fig. 3. Light microscopy images (7.5 $\mathrm{mm}$ width and $5.5 \mathrm{~mm}$ height) of $0.5 \mathrm{~mm}$ thick samples from each preparation method (rows) and with different nanotube concentrations (columns).

\section{Theory}

In this chapter we derive an extremely simplified model of percolated particles in order to try to relate the sample conductivity (that can be measured) to the inter-particle contact resistance inside the polymer (that cannot be measured, but ultimately limits charge transport). The model describes homogenously dispersed, immobile particles that are presumed to be rigid and of cylindric shape. The network they build is modeled through $n$ parallel paths bridging the sample, each consisting of $m$ sticks and $m$ contacts. The number of parallel connections of single paths bridging the sample is defined as

$$
n=\frac{V_{\text {all }}}{m \cdot V_{\text {one }}}=\frac{\Phi_{\text {vol }} \cdot V_{\text {sample }}}{m \cdot V_{\text {one }}} \approx \frac{\rho_{\text {matrix }} \Phi_{w t}}{\rho_{\text {all }}} \cdot \frac{V_{\text {sample }}}{m \cdot V_{\text {one }}}
$$

where the sample volume $V_{\text {sample }}$ completely vanishes in the subsequent calculations so that we have to deal with the single particle volume $V_{\text {one }}$ only. For low loading fractions 
the filler volume concentration $\Phi_{v o l}$ can be approximated with the filler weight concentration $\Phi_{w t}$ (as only controllable parameter in our experiments, we always denote the filler weight fraction without index) using the matrix density $\rho_{\text {matrix }}$ and filler density $\rho_{\text {all }}$. All filler to matrix density ratios encountered in this study as well as in the references analyzed in the next section are around 2:1. The number of particles and inter-particle contacts within a single path is conveniently defined as

$$
m=\frac{t}{l} \cdot \frac{1}{\Phi^{x}}
$$

for being proportional to the sample thickness $t$ and inversely proportional to the particle length $l$ and filler weight fraction $\Phi$. The latter dependence expresses the fact that all particles that experimentally build dead paths are theoretically incorporated in the conducting ones which makes these paths longer. The tendency to have dead paths is bigger for lower filler concentrations, therefore $m$ increases with decreasing filler concentration. The exponential dependence is solely motivated by the experimentally found power law above 0.1 wt\% (blue, dashed line in Fig. 2). Therefore, the magnitude of the exponent $x$ will always be adjusted according to the respective experimental results. Setting up the equations for parallel and series resistances within a sample yield the relation

$$
\sigma_{\text {Sample }} \approx \frac{l}{2 \pi \cdot r^{2}} \cdot \frac{\Phi^{2 x+1}}{R+R_{C}}
$$

where $r$ and $R$ are the radius and resistance of a single particle, respectively, and $R_{C}$ is the resistance of its contact to the next particle. In short, the recipe for assessing $R_{C}$ consists of equating $2 x+1$ with the slope of the $\log \sigma_{\text {Sample }}$ vs $\log \Phi$ plot above the percolation threshold and then solving Eq. (3) with the help of the known parameters: individual particle size and resistance as well as conductivity and concentration values corresponding to a single data point.

\section{Discussion}

\subsection{Two network formation mechanisms and percolation thresholds}

We believe that the existence of two types of percolation thresholds is a characteristic feature of composite materials that possess a fluid state of low viscosity during processing. The higher threshold is determined by statistical percolation theory and therefore is unchangeable by processing methods. The lower one can vastly be shifted down to lower concentrations by stimulating particle flocculation and network formation.

Evidence for our statement is provided through comparison of the conductivity measurements in Fig. 2 with the light microscopy images in Fig. 3. Both, the emergence of flocs and the steep conductivity increase, occur at lower filler concentrations than predicted by percolation theory for statistically distributed particles. This theoretical value 
represents the lower limit where a conducting network develops due to contacts between homogenously dispersed, immobile particles. We assume that at this point flocculation cannot further improve the conductivity or that particle rearrangement even cannot occur due to lowered particle mobility through the established network. Right below this point the superstructure of flocs is able to retain a certain level of conductivity (plateau) and above this point conductivity increases solely due to the generation of additional paths that connect opposite sample sites (power law dependence). This point at $0.1 \mathrm{wt} \%$ (arrow in Fig. 2) represents in our opinion the real statistical percolation threshold, whereas the additional thresholds at lower concentrations $(0.011,0.024$ and $0.08 \mathrm{wt} \%$ ) are of dynamic origin. The kinetic percolation threshold of the FS-samples at $0.08 w t \%$ thereby overlaps the statistical threshold at $0.1 \mathrm{wt} \%$.

Now we want to analyze which mechanism is responsible for the different flocculation intensities for the SS-, MS- and FS-samples. Diffusion, convection, van der Waals and Coulomb forces can be excluded as they are present in all samples and should initiate identical flocculation all-over. The only difference arises from the variation of the stirring rates and temperatures after hardener addition. Thus, shear forces at low viscosities seem to be the only promoter of flocculation, at least regarding the short time period (1 hour) until polymer gelation. The significance of shearing was already demonstrated in experiments [20] as well as in simulations [21], where Klingenberg et al. further predict a strong dependence of flocculation on the stiffness and shape of the filler particles and the friction forces between them.

\subsection{Charge transport through the nanotube network}

Statistical percolation theory does not directly describe the conductivity increase due to the percolation behavior of conductive particles, but merely the amount of particles of a given size needed to form infinite clusters of particles which are in contact with each other. Whether the particle size includes an insulating layer as well as its possible thickness finally determines the magnitude of conductivity improvement through percolation.

In this context conductivity below the percolation threshold is sometimes explained with tunneling conduction between homogeneously distributed particles not yet in physical contact (yielding the proportionality $\log \sigma_{\text {Sample }} \sim \Phi^{-1 / 3}$ ) [18,22]. This argument is not valid in our case as here all homogeneity is lost while lowering the threshold by as much as a factor of 10. Nevertheless, the corresponding plot of our results (Fig. 4) is worth to be discussed. All samples show above $0.2 \mathrm{wt} \%$ the mentioned proportionality which is however lost below $0.1 \mathrm{wt} \%$. While this result suggests tunneling conduction rather above statistical percolation threshold than below, we want to emphasize possible limitations of this analysis. First of all, it is not clear whether a deviation from the given proportionality indicates a change in the conduction mechanism or simply the transition 
to an inhomogeneous particle distribution. Moreover, the plots of $\log \sigma_{\text {Sample }}$ vs $\Phi^{-1 / 2}$ or $\Phi^{-1 / 4}$ yield a comparable linear fit.

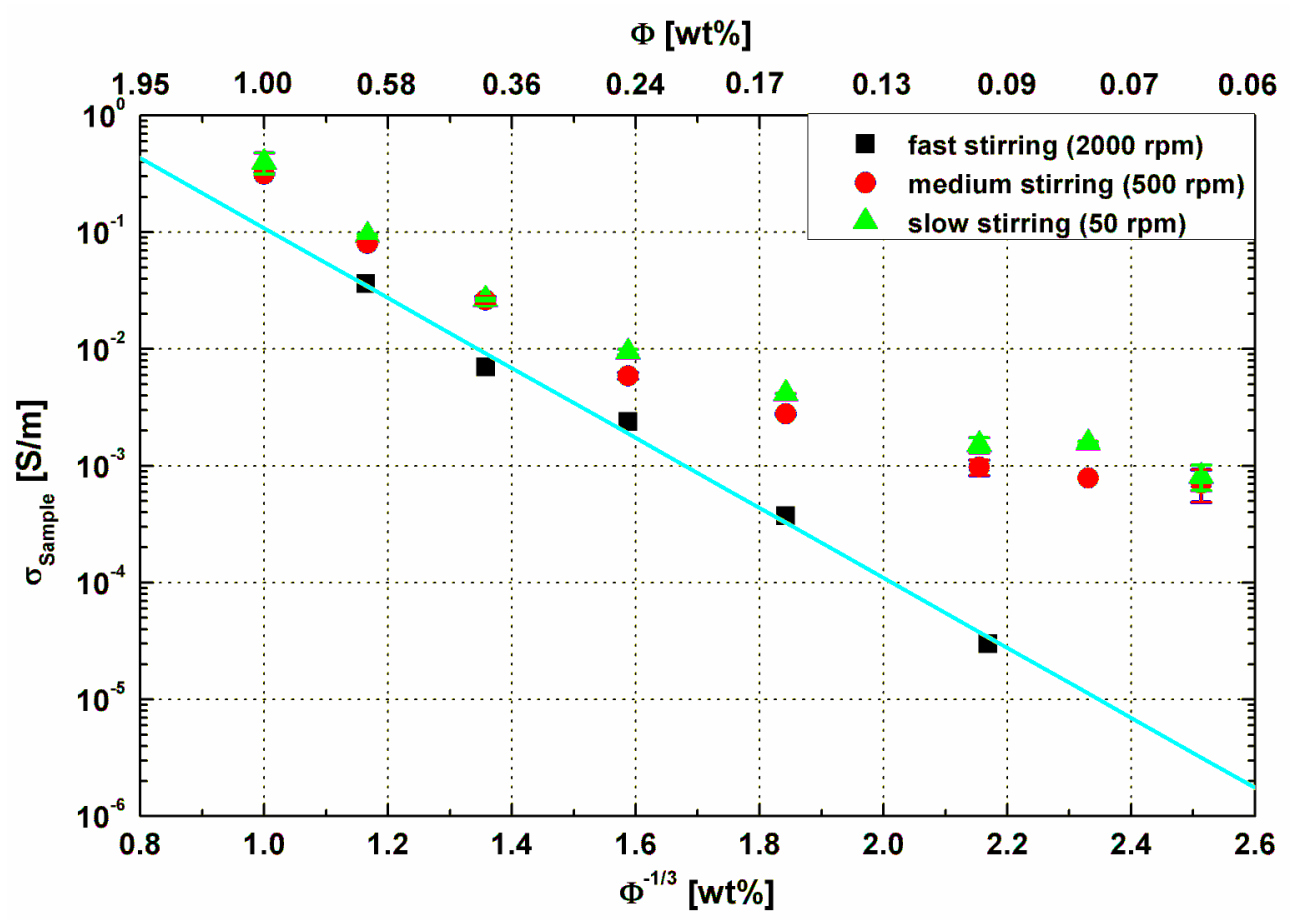

Fig. 4. Logarithmic plot of conductivity vs $\Phi^{-1 / 3}$. A linear dependence indicates tunnelling transport of charge carriers.

\subsection{Inter-particle contact resistances of various composite materials}

We start with explaining in detail how we proceeded in assessing the inter-particle contact resistances from the data of other groups and from our results. It is obvious that the only parameter needed besides the conductivity results is the size of an individual filler particle inside the polymer. The particle size highly depends on the sample processing methods and is not necessarily equal to the pristine filler size. Therefore, a crosscheck is necessary to compare the critical concentration predicted by percolation theory for the stated filler size [8] with the experimentally determined threshold. In the majority of cases the length of the particles was adjusted to fit the theoretical predictions, while mostly staying within the length distribution stated by the authors. The exponential dependence of $\sigma_{\text {Sample }}$ on $\Phi$ was subsequently determined from the slope of the respective log-log plot above the percolation threshold. Conductivity and concentration values corresponding to a single data point were extracted from the same region and finally the contact resistance magnitude was determined.

The above calculations were applied to conductivity data presented in this work as well as to published data that provided both, information about their filler sizes and some conductivity values above their statistical percolation threshold. The first striking result of this analysis is the fact that except for one case all slopes of the log-log plots above the 
percolation threshold reveal similar values around 3 . In addition, this statement also holds for all our three preparation methods above $0.1 \mathrm{wt} \%$ (Fig. 2) thus suggesting a kind of universality in the nature of the network above the statistical percolation threshold.

Table 1. Summary of the experimental parameters of conductivity measurements found in literature with the resultant values needed for assessing the contact resistance $R_{C}$. Asterisks denote statistical percolation thresholds that had been derived from the filler sizes because the thresholds found experimentally were too low and thus presumably of dynamic type. Question marks indicate particle sizes that had been guessed (according to the percolation threshold) due to the lack of sufficient information.

\begin{tabular}{|c|c|c|c|c|c|c|c|}
\hline Filler & Matrix & Preparation method & $\Phi_{C}$ & $l / d$ & $2 x+1$ & $\boldsymbol{R}_{C}$ & Ref. \\
\hline MWNT (CVD) & PC & $\begin{array}{c}\text { Extruded and } \\
\text { compression moulded }\end{array}$ & 1.4 wt $\%$ & $100=\frac{1 \mu \mathrm{m}}{10 \mathrm{~nm}}$ & 3.1 & $10^{5} \Omega$ & [11] \\
\hline $\begin{array}{c}\text { MWCNT } \\
\text { (Arc discharge) }\end{array}$ & PmPV & $\begin{array}{l}\text { Filler and matrix } \\
\text { sonicated in toluene }\end{array}$ & $7.5 w t \%$ & $20=\frac{400 \mathrm{~nm}}{20 \mathrm{~nm}}$ & 5.3 & $10^{7} \Omega$ & [12] \\
\hline MWNT (CVD) & Epoxy & $\begin{array}{l}\text { Filler dispersed in methanol, } \\
\text { composite cured under pressure }\end{array}$ & $0.7 w t \%$ & $250=\frac{25 \mu \mathrm{m} ?}{100 \mathrm{~nm}}$ & 3.1 & $10^{3} \Omega$ & [13] \\
\hline $\begin{array}{l}\text { PPy coated } \\
\text { cellulose }\end{array}$ & Latex & Stirred, freeze dried, moulded & 5 vol\% * & $12.5=\frac{2 \mu \mathrm{m}}{160 \mathrm{~nm}}$ & 2.8 & $10^{6} \Omega$ & [14] \\
\hline $\begin{array}{c}\text { SW- and } \\
\text { DWCNT (CCVD) }\end{array}$ & Epoxy & $\begin{array}{c}\text { Filler dispersed in water, } \\
\text { composite cured in teflon mould }\end{array}$ & $0.08 w t \%$ & $2000=\frac{6 \mu \mathrm{m} ?}{3 \mathrm{~nm}}$ & 3.3 & $10^{5} \Omega$ & [15] \\
\hline MWCNT (CCVD) & Epoxy & Stirred, cured in metal mould & $0.1 \mathrm{wt} \%$ * & $1000=\frac{15 \mu \mathrm{m} ?}{15 \mathrm{~nm}}$ & 2.7 & $10^{5} \Omega$ & $\begin{array}{l}\text { This } \\
\text { work }\end{array}$ \\
\hline
\end{tabular}

All relevant parameters and the resulting contact resistance magnitudes are summarized in Table 1. Two of the analyzed publications $[13,14]$ yield particle contact resistances of the same magnitude as the resistances of the individual filler particles respectively used, namely $6.4 \mathrm{k} \Omega$ [23] for a single multi-wall nanotube and some $M \Omega$ [14] for a pure PPy coated cellulose particle. These contact resistances are the lowest computable results as both summands in the denominator of Eq. (3) are of same magnitude and small parameter variations easily decrease the denominator below $R$ thus leading to negative values of $R_{C}$. Therefore, straight-lined filler particles with big diameters seem to give low contact resistances, no matter what type of filler, matrix or processing method is used. The second set of published data $[11,15]$ as well as our work exhibit a contact resistance in the region of $10^{5} \Omega$, thus 100 times larger than the pure single-wall [24] or multi-wall nanotube resistance. Again, neither the type of nanotubes or polymer nor the processing method seem to have a noticeable impact on the contact resistance magnitude. Finally, the high contact resistance in the case of the PmPV matrix [12] can be explained with the helical structure of the polymer chains which presumably wrap the nanotubes and having a diameter of $2 \mathrm{~nm}$ - impedes electron tunneling between them. 


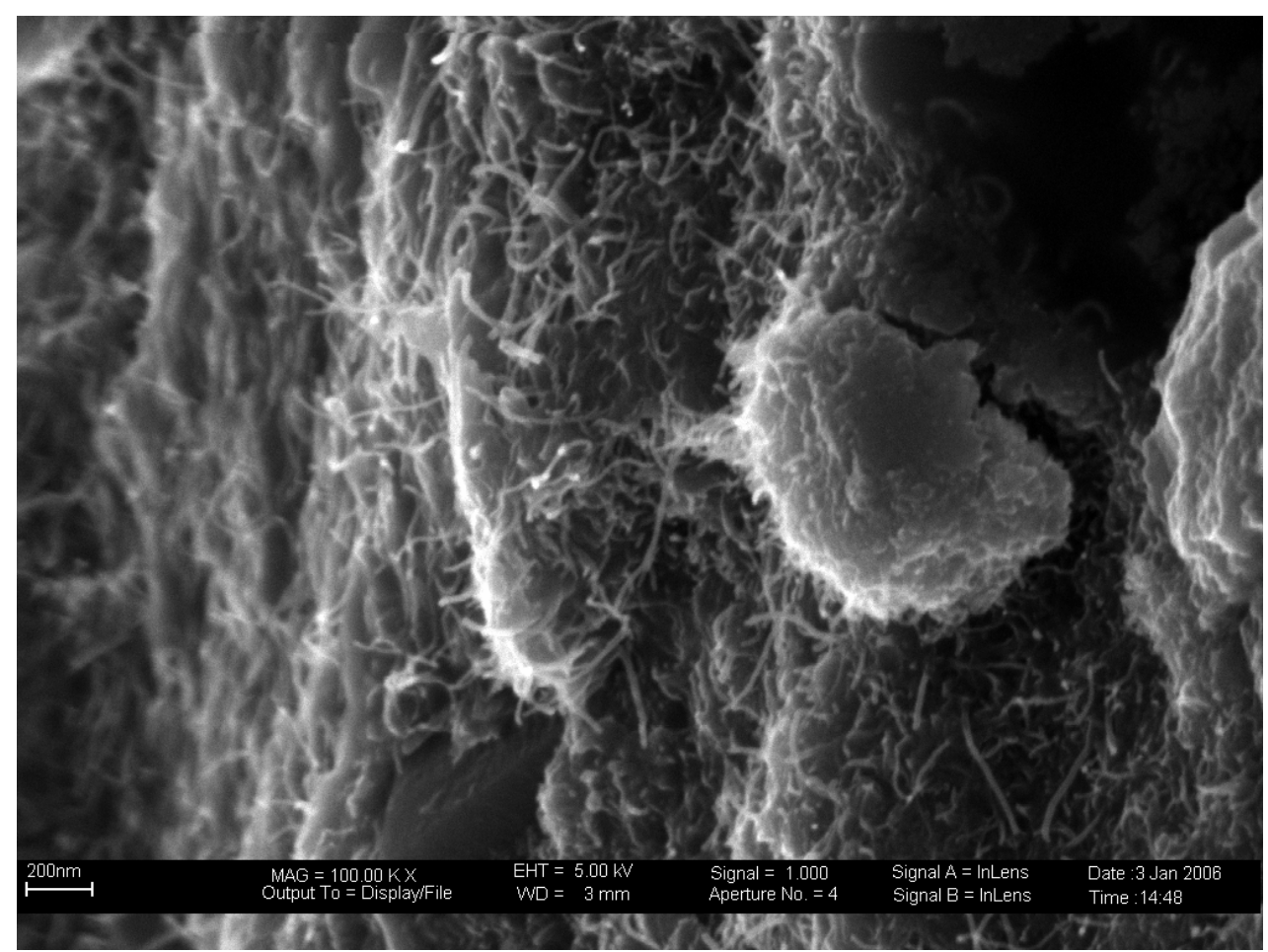

Fig. 5. SEM-image of the fracture surface of a zone of very high local nanotube concentration within a 1 wt\%-sample. The scale bar is $200 \mathrm{~nm}$.

One reason for the restricted possibility of data comparison definitely is the simplicity of our model, which does not include the real state of the dispersion, eg the magnitude of particle agglomerates, the degree of particle wetting through the matrix and the real particle shape. However, including these parameters into our model simultaneously requires the publication of corresponding SEM and TEM analyses to provide these data. In our case, SEM-images of fracture surfaces reveal zones of very high local nanotube concentration with diameters up to $100 \mu \mathrm{m}$. It is clearly visible that the nanotubes within these zones are not bundled but have a curly shape (Fig. 5). With an improved model and enough data points above the respective statistical percolation thresholds it should be possible to analyze the dependence on different filler particles, matrices and preparation methods. This is the only way to both, supply more and reliable parameters - along with the particle sizes - and verify the universality of the linear dependence stated in this study.

\section{Conclusions}

Conductivity measurements over two decades of filler concentration with narrow increments revealed two percolation thresholds, the lower one attributed to a kinetic and the higher one to a static network formation process. A conductivity plateau was detected in our results and was attributed to the presence of a superstructure of flocculated filler particles. The onset of this flocculation was controlled by coarsely adjusting the stirring rate and temperature during the final mixing step. Qualitative investigations on these parameters with a rotational rheometer - allowing simultaneous optical and electrical measurements - are under way. 
The flocculation generates a percolation threshold that is no longer explained with statistical percolation theory - colloid theory needs to be applied instead. Nevertheless, the crossover from saturation to power law behavior at $0.1 \mathrm{wt} \%$ coincides with the predicted statistical percolation threshold. Thus, attention has to be paid to the validity regions of the different theories when modeling percolation behavior.

A simple geometrical model was developed to fit the power law dependence of conductivity above the statistical percolation threshold and to derive the magnitude of an individual inter-particle contact resistance. The analysis of appropriate published data could not reveal a dependence on the filler or matrix type, thus necessitating further experiments with various materials and more data points. The high contact resistance expected for the case of the nanotubes wrapped by polymer structures was reproduced by our model. Medium contact resistances were obtained for nanotubes with diameters of several $\mathrm{nm}$ and lengths of several $\mu \mathrm{m}$. Straight-lined filler particles with diameters of around $100 \mathrm{~nm}$ seem to yield rather low resistances. Extensive sample characterizations as well as thorough model refinements are however necessary to give more detailed statements.

\section{Acknowledgements}

The authors wish to thank Dr. Ron Schmidt of the University of Cambridge for valuable comments and Huntsman Advanced Materials for providing the epoxy resin. The European Thematic Network 'CNT-Net' [G5RT-CT-2001-05026] is acknowledged for providing several opportunities for discussions within the Network. 
[1] Saar MO, Manga M. Continuum percolation for randomly oriented soft-core prisms. Phys Rev E 2002;65(5):56131.

[2] Youngs IJ. A geometric percolation model for non-spherical excluded volumes. J Phys D 2003;36(6):738-47.

[3] Celzard A, McRae E, Deleuze C, Dufort M, Furdin G, Marêché JF. Critical concentration in percolating systems containing a high-aspect-ratio filler. Phys Rev B 1996;53(10):6209-14.

[4] Balberg I, Anderson CH, Alexander S, Wagner N. Excluded volume and its relation to the onset of percolation. Phys Rev B 1984;30(7):3933-43.

[5] Natsuki T, Endo M, Takahashi T. Percolation study of orientated short-fiber composites by a continuum model. Physica A 2005;352(2-4):498-508.

[6] Sandler J, Shaffer MSP, Prasse T, Bauhofer W, Schulte K, Windle AH. Development of a dispersion process for carbon nanotubes in an epoxy matrix and the resulting electrical properties. Polymer 1999;40(21):5967-71.

[7] Sandler JKW, Kirk JE, Kinloch IA, Shaffer MSP, Windle AH. Ultra-low electrical percolation threshold in carbon-nanotube-epoxy composites. Polymer 2003;44(19):5893-9.

[8] Martin CA, Sandler JKW, Shaffer MSP, Schwarz MK, Bauhofer W, Schulte K, Windle AH. Formation of percolating networks in multi-wall carbon-nanotube-epoxy composites. Comp Sci Tech 2004;64(15):230916.

[9] Martin CA, Sandler JKW, Windle AH, Schwarz MK, Bauhofer W, Schulte K, Shaffer MSP. Electric fieldinduced aligned multi-wall carbon nanotube networks in epoxy composites. Polymer 2005;46(3):877-86.

[10] Van de Ven TGM. Colloidal hydrodynamics. London: Academic Press, 1989.

[11] Poetschke P, Dudkin SM, Alig I. Dielectric spectroscopy on melt processed polycarbonate-multiwalled carbon nanotube composites. Polymer 2003;44(17):5023-30.

[12] Curran SA, Ajayan PM, Blau WJ, Carroll DL, Coleman JN, Dalton AB, Davey AP, Drury A, McCarthy B, Maier S, Strevens A. A Composite from Poly (m-phenylenevinyleneco-2,5-dioctoxy-pphenylenevinylene) and Carbon Nanotubes: A Novel Material for Molecular Optoelectronics. Adv Mat 1998;10(14):1091-93.

[13] Allaoui A, Bai S, Cheng HM, Bai JB. Mechanical and electrical properties of a MWNT/epoxy composite. Comp Sci Tech 2002;62(15):1993-8.

[14] Flandin L, Bidan G, Brechet Y, Cavaille JY. New Nanocomposite Materials Made of an Insulating Matrix and Conducting Fillers: Processing and Properties. Polym Comp 2000;21(2):165-74.

[15] Barrau S, Demont P, Maraval C, Bernes A, Lacabanne C. Glass Transition Temperature Depression at the Percolation Threshold in Carbon Nanotube-Epoxy Resin and Polypyrrole-Epoxy Resin Composites. Macrom R Comm 2005;26(5):390-4.

[16] Kirkpatrick S. Percolation and Conduction. Rev Mod Phys 1973;45(4):574-88.

[17] Stauffer D, Aharony A. Introduction to percolation theory. London: Taylor and Francis, 1992.

[18] Kilbride BE, Coleman JN, Fraysse J, Fournet P, Cadek M, Drury A, Hutzler S, Roth S, Blau WJ.

Experimental observation of scaling laws for alternating current and direct current conductivity in polymercarbon nanotube composite thin films. J Appl Phys 2002;92(7):4024-30.

[19] Bryning MB, Islam MF, Kikkawa JM, Yodh AG. Very Low Conductivity Threshold in Bulk Isotropic Single-Walled Carbon Nanotube-Epoxy Composites. Adv Mat 2005;17(9):1186-91.

[20] Lin-Gibson S, Pathak JA, Grulke EA, Wang H, Hobbie EK. Elastic Flow Instability in Nanotube Suspensions. Phys Rev Lett 2004;92(4):048302.

[21] Switzer LH, Klingenberg DJ. Flocculation in simulations of sheared fiber suspensions. Int J Multiphase Flow 2004;30(1):67-87.

[22] Ezquerra TA, Kulescza M, Santa Cruz C, Baltá-Calleja FJ. Charge Transport in PolyethyleneGraphite Composite Materials. Adv Mat 1990;2(12):597-600.

[23] Sanvito S, Kwon YK, Tománek D, Lambert CJ. Fractional Quantum Conductance in Carbon Nanotubes. Phys Rev Lett 2000;84(9):1974-7.

[24] Gao B, Chen YF, Fuhrer MS, Glattli DC, Bachtold A. Four-Point Resistance of Individual Single-Wall Carbon Nanotubes. Phys Rev Lett 2005;95(19):196802. 